\title{
A Case Report of Lemierre's Syndrome with Multisystem Dysfunction in the Setting of COVID-19 Exposure in a Pediatric Patient
}

\section{Kathleen McMahon ${ }^{1 *}$, Odiraa Nwankwor ${ }^{2}$, Christina Palladino ${ }^{2}$, Laura Czulada ${ }^{2}$, Cameron Jacobs ${ }^{1}$}

${ }^{1}$ Department of Emergency Medicine, St. Luke's University Health Network, Bethlehem, PA, USA

${ }^{2}$ Department of Pediatric Critical Care, St. Luke's University Health Network, Bethlehem, PA, USA

Email: ${ }^{\star K m}$ cmahon94@gmail.com

How to cite this paper: McMahon, K., Nwankwor, O., Palladino, C., Czulada, L. and Jacobs, C. (2021) A Case Report of Lemierre's Syndrome with Multisystem Dysfunction in the Setting of COVID-19 Exposure in a Pediatric Patient. Case Reports in Clinical Medicine, 10, 246-251.

https://doi.org/10.4236/crcm.2021.109032

Received: August 20, 2021

Accepted: September 24, 2021

Published: September 27, 2021

Copyright $\odot 2021$ by author(s) and Scientific Research Publishing Inc. This work is licensed under the Creative Commons Attribution International License (CC BY 4.0).

http://creativecommons.org/licenses/by/4.0/

\begin{abstract}
A 17-year-old male with no significant past medical history presented to the emergency department with severe sepsis of unknown etiology. The patient was found to have septic thrombophlebitis of the internal jugular vein with multiorgan dysfunction and septic embolization to both lungs. The patient was also noted to have COVID-19 IgM antibodies and multiple close COVID-19 exposures prior to the patient's emergency department presentation. Here, we present the prolonged and complicated hospitalization of this patient and a review of this rare but important disease.
\end{abstract}

\section{Keywords}

Lemierre's Syndrome, Sepsis, COVID-19, Thrombophlebitis, Septic Embolism

\section{Introduction}

Lemierre's syndrome, a superficial thrombophlebitis of the internal jugular vein, has been noted in pediatric patients with increasing frequency over the last few decades [1]. One theory for the disease's re-emergence is the decreased frequency of antibiotic therapy for general pharyngitis [2]. This condition is associated with significant morbidity and mortality secondary to septic shock, embolization of infected clot throughout the body, and vascular occlusion. For this reason, it is critical to make an accurate diagnosis and to initiate treatment expeditiously. Unfortunately, given the relative rarity of the condition and broad differential diagnosis, there is often a delay in identification [2]. Here, we present a case of 
an adolescent with Lemierre's syndrome with significant multisystem complications and prolonged hospital course.

\section{Case Presentation}

A 17-year-old male without significant past medical history presented to the Emergency Department (ED) with complaints of 5 days of cough, fever, myalgias, nausea, vomiting, and shortness of breath in the setting of recent close contact with a case of COVID-19. The patient was tachycardic but otherwise had an unremarkable physical exam. He was subsequently discharged from the ED with diagnosis of presumed COVID-19 infection with close return precautions.

The patient returned to the ED two days later with shortness of breath, generalized abdominal pain in addition to worsening of his initial symptoms. He also complained of severe throat pain. He was tachycardic, mildly hypotensive, and required nasal cannula oxygenation for hypoxia. He had dry mucous membranes, bilateral anterior cervical adenopathy, increased work of breathing, and generalized abdominal tenderness with guarding. The patient's initial workup showed hyponatremia, acute kidney injury (AKI), severe thrombocytopenia and increased inflammatory markers. See Table 1 for abnormal laboratory results. Initial COVID-19 PCR was negative. AST and ALT were within normal limits.

A non-contrast CT of soft tissue neck, chest, abdomen, and pelvis was performed due to AKI and showed diffuse bilateral consolidations in the lungs. He had patchy bilateral consolidations on chest $\mathrm{x}$-ray.

The patient was admitted to the pediatric intensive care unit for management of shock and multiorgan dysfunction. He was intubated for acute hypoxic respiratory failure and required norepinephrine briefly for fluid refractory distributive shock. He later developed large bilateral loculated pleural effusions requiring bilateral chest tube placement. CTA of the neck, performed after resolution of AKI, showed a right $21 \mathrm{~mm}$ peritonsillar abscess with focal blood clot in the right internal jugular vein, consistent with Lemierre's syndrome (Figure 1). CT of the chest demonstrated bilateral septic emboli (Figure 2). Two echocardiograms performed during admission were negative for vegetations. He was treated empirically for sepsis with cefepime, metronidazole, and vancomycin, but therapy was later narrowed to ampicillin-sulbactam once blood cultures grew Fusobacterium necrophorum, Eikenella corrodens, and Streptococcus constellatus. He was ultimately placed ceftriaxone and Flagyl to complete a 6-week course of therapy.

During his prolonged and complicated hospital stay, he had significant thrombocytopenia, not associated with disseminated intravascular coagulation or a hemolytic process. Early in his stay he received one dose of IVIG due to concern for possible thrombotic thrombocytopenic purpura. Subsequently his platelet count improved. He received multiple blood transfusions for persistent anemia. He was treated with enoxaparin and acetylsalicylic acid $81 \mathrm{mg}$ after follow-up imaging noted extension of the internal jugular vein clot and new 
Table 1. Initial patient laboratory studies.

\begin{tabular}{cc}
\hline Test & Result \\
Sodium & $128 \mathrm{mmol} / \mathrm{L}$ \\
BUN & $94 \mathrm{mmol} / \mathrm{L}$ \\
Creatinine & $4.51 \mathrm{mmol} / \mathrm{L}$ \\
Albumin & $1.8 \mathrm{~g} / \mathrm{dL}$ \\
D-dimer & $2.76 \mathrm{ug} / \mathrm{mL} \mathrm{FEU}$ \\
Lactate & $2.5 \mathrm{mmol} / \mathrm{L}$ \\
WBC & 19.49 thousand $/ \mathrm{uL}$ \\
Hemoglobin & $11.6 \mathrm{~g} / \mathrm{dL}$ \\
Platelets & 12 thousand/uL \\
NT pro-BNP & $22,530 \mathrm{pg} / \mathrm{mL}$ \\
CRP & $305.5 \mathrm{mg} / \mathrm{L}$ \\
Troponin & $0.07 \mathrm{ng} / \mathrm{mL}$ \\
SARS-CoV IgM & Positive
\end{tabular}

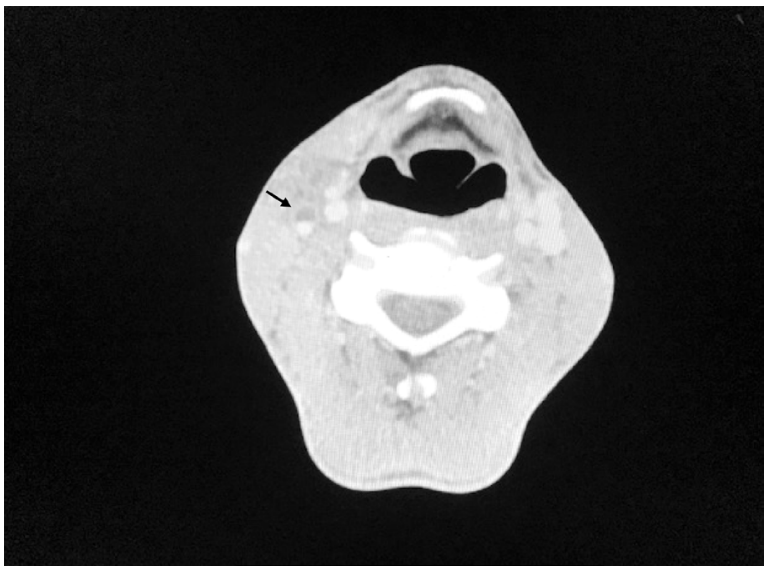

Figure 1. CTA of the head and neck demonstrating thrombus in the right internal jugular vein (black arrow) with surrounding soft tissue edema.

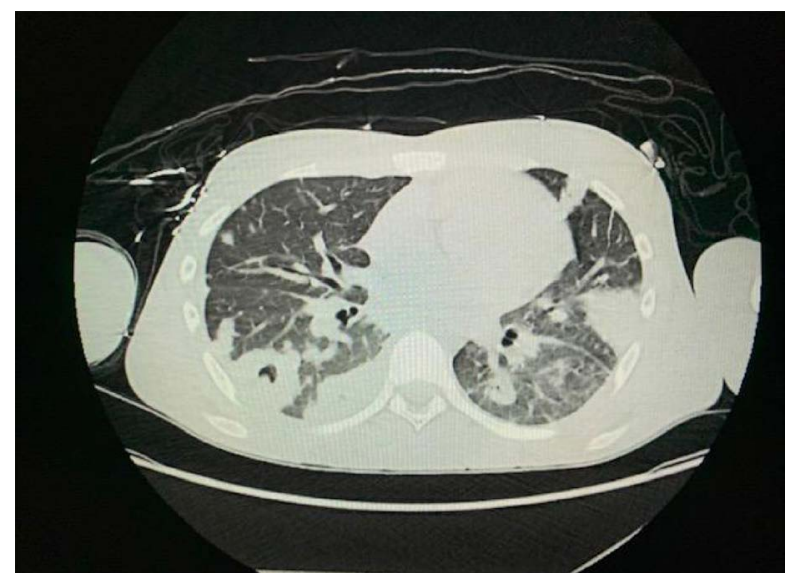

Figure 2. CT of the chest with contrast demonstrating multifocal consolidations, some with cavitation, consistent with septic emboli. 
cavernous sinus thrombosis. He also was noted to have new right cranial nerve 12 palsy which was managed with anticoagulation therapy. Though considered for surgical ligation of the involved vessel, this measure was not ultimately required.

The patient was discharged to home to complete a 6-week course of IV antibiotics. At the time of discharge he had been weaned from supplemental oxygen and his cranial nerve palsy was improving. The weight loss and malnutrition that developed over the course of his critical illness and prolonged ICU stay slowly improved by the time of discharge.

\section{Discussion}

Lemierre's syndrome was first described by Andre Lemierre in 1936. Prior to the widespread introduction of penicillin, the frequency and mortality of the disease were much higher than today [2]. Lemierre's syndrome classically originates from an ipsilateral oropharyngeal infection such as tonsillitis or peritonsillar abscess. The implicated bacteria are most commonly gram-negative anaerobes. It is thought that bacteria may gain entry to nearby vasculature due to disruption in normal mucosal integrity associated with oropharyngeal infection or via traumatic inoculation [3]. Fusobacterium necrophorum is the most commonly isolated pathogen, though other gram-negative anaerobes and anaerobic streptococci may also be identified. One third of cases are polymicrobial [4]. Clot extension to facial veins, dural venous sinuses, or the cavernous sinus may occur. Embolization of the clot to distant sites is frequent.

\subsection{Diagnosis}

Diagnosis begins with clinical suspicion of the disease. Physical exam findings consistent with the diagnosis include sore throat, high fever, rigors, neck pain, dry cough, pleuritic chest pain, and exam findings consistent with septic embolization [2]. Identification of the septic thrombus is via imaging. Ultrasound, CT scan, or MRI may be performed, with CT of the neck with contrast the study of choice [5]. Blood cultures aid in diagnosis and in identification of the causative organism(s), which guides antimicrobial selection.

Having a high index of suspicion for the disease is paramount given the expansive differential diagnosis for the classic presenting symptoms. A recent case report details a case similar to ours, in which a 15-year-old female developed sepsis with multiorgan failure due to Lemierre's disease. Our subject exhibited significant pulmonary involvement and was initially thought to be due to possible MIS-C given high prevalence of COVID-19 in the patient's community and his positive COVID antibody tests. Given the current COVID-19 pandemic, it is even more important to consider Lemierre's syndrome on the differential for pediatric neck pain, pharyngitis, viral syndrome and acute hypoxic respiratory failure to prevent misdiagnosis [6].

\subsection{Treatment}

Antibiotic therapy is the cornerstone of treatment. Antibiotic choices cited in the 
literature include carbapenems, beta-lactam/beta-lactamase inhibitor, ceftriaxone, or clindamycin; these may be used with or without metronidazole. It is recommended to initiate broad spectrum antibiotics pending culture and sensitivity results [7]. The duration of therapy is typically $3-6$ weeks.

The use of anticoagulation is controversial. In one study, $21 \%-64 \%$ of patients with the disease received anticoagulation but no clear benefit was demonstrated [2] [7]. Recent studies have shown that anticoagulation may not impact outcomes associated with thrombosis [8] [9]. Our patient was placed on anticoagulation when repeat imaging demonstrated clot extension into the cavernous sinus and a new neurologic deficit. This is consistent with certain expert recommendations that anticoagulation may be beneficial in those cases with retrograde clot extension into the cavernous sinus [2].

Surgical intervention is uncommon. In unusual cases, ligation of the internal jugular vein is performed, typically in critically ill patients with the goal to prevent further embolization.

\subsection{Complications}

This disease has significant morbidity and mortality. While wide embolization of the clot can occur, the lungs are the most common location [4]. Septic emboli may also be seen in the intra-abdominal viscera, joints, soft tissue, and the brain. One case series of nine children with Lemierre's syndrome demonstrated five cases of septic pulmonary emboli and two cases of septic shock. Long-standing adverse effects of this disease are often due to septic emboli and organ dysfunction that may result from them. While diffuse abdominal pain is described as a symptom of this disease, the precise etiology in our particular patient is unknown. Imaging of the abdomen was unremarkable. It is possible that he had referred pain due to a significant burden of septic pulmonary emboli.

\subsection{Prognosis}

Mortality rates for all age groups range from 0 - 18\% with proper treatment. For survivors, significant morbidity related to complications such as vascular occlusion, embolic phenomena, and prolonged hospitalization may result.

\section{Conclusion}

This is a case of Lemierre's syndrome in a previously healthy 17 -year-old male with COVID-19 exposure who developed multi-organ dysfunction syndrome. He required mechanical ventilation and bilateral tube thoracostomies for severe pulmonary involvement. Other rare features of the case included severe thrombocytopenia and anemia, acute renal failure, cavernous sinus thrombosis, and isolated cranial nerve 12 palsy. To our knowledge, this is the first case of Lemmierre's Syndrome in a pediatric patient with COVID-19 antibodies. The importance of early diagnosis and treatment may be lifesaving, and it is important to consider this syndrome when evaluating pediatric patients with neck pain and 
acute respiratory failure in this era of COVID-19 pandemic.

\section{Conflicts of Interest}

The authors declare no conflicts of interest regarding the publication of this paper.

\section{References}

[1] Goldenberg, N.A. (2005) Lemierre's and Lemierre's-Like Syndromes in Children: Survival and Thromboembolic Outcomes. Pediatrics, 116, e543-e548. https://doi.org/10.1542/peds.2005-0433

[2] Riordan, T. (2004) Lemierre's Syndrome: More than a Historical Curiosa. Postgraduate Medical Journal, 80, 328-334. https://doi.org/10.1136/pgmj.2003.014274

[3] Srivali, N., Ungprasert, P., Kittanamongkolchai, W. and Ammannagari, N. (2014) Lemierre's Syndrome: An Often Missed Life-Threatening Infection. Indian Journal of Critical Care Medicine, 18, 170-172. https://doi.org/10.4103/0972-5229.128708

[4] Rafati, F., Bhalla, V., Chillal, V. and Thyagarajan, M. (2017) Lemierre Syndrome: A Diagnostic Dilemma in Paediatric Patients. Journal of Surgical Case Reports, 2017, rjx210. https://doi.org/10.1093/jscr/rjx210

[5] Kushawaha, A., Popalzai, M., El-Charabaty, E. and Mobarakai, N. (2009) Lemierre's Syndrome, Reemergence of a Forgotten Disease: A Case Report. Cases Journal, 2, No. 1. https://doi.org/10.1186/1757-1627-2-6397

[6] Repper, D.C., Arrieta, A.C., Cook, J.E. and Renella, P. (2020) A Case of Lemierre Syndrome in the Era of COVID-19: All That Glitters Is Not Gold. The Pediatric Infectious Disease Journal, 39, e445-e447. https://doi.org/10.1097/INF.0000000000002939

[7] Johannesen, K. and Bodtger, U. (2016) Lemierre's Syndrome: Current Perspectives on Diagnosis and Management. Infection and Drug Resistance, 9, 221-227. https://doi.org/10.2147/IDR.S95050

[8] Adedeji, A., Chukwura, O., Obafemi, T., McNulty, S.B. and Reinert, J.P. (2020) Anticoagulation Strategies in the Management of Lemierre Syndrome: A Systematic Review of the Literature. Annals of Pharmacotherapy, 55, 658-665. https://doi.org/10.1177/1060028020957620

[9] Cupit, M.C., Nageswara Rao, A., Warad, D.M., Khan, S. and Rodriguez, V. (2015) Lemierre's Syndrome: Role of Anticoagulation and Thrombosis Outcomes, a Retrospective Study. Blood, 126, 2296.

https://doi.org/10.1182/blood.V126.23.2296.2296 